\title{
Sobre el pensamiento feminista y la ciencia
}

\author{
On the Feminist Thought and Science
}

\author{
Teresa Arrieta de Guzmán \\ Universidad Nacional de San Agustín, Arequipa, Perú \\ Contacto:darrieta@unsa.edu.pe \\ https://orcid.org/0000-0003-2384-2721
}

\begin{abstract}
Resumen
En este artículo trataremos de la relación del feminismo con la ciencia. Primero, ofreceremos una visión panorámica de las diversas posiciones que se han dado respecto del pensamiento feminista y su aporte a los campos de la Teoría del conocimiento y de la Filosofía de la ciencia. Luego, presentaremos las doctrinas de Sandra Harding y Susan Haack, que muestran la polarización entre posiciones que rechazan totalmente la concepción y práctica de la ciencia pasada y presente por considerarla absolutamente androcéntrica, frente a quienes sostienen que la ciencia no tiene nada que ver con el género, resultando por lo tanto incongruente la sola idea de una epistemología feminista. Finalmente, haremos un análisis de lo expuesto, reafirmando conceptos que consideramos realistas y rechazando los que juzgamos erróneos o exagerados, para llegar a nuestras propias conclusiones, incidiendo en la importancia de no soslayar puntos de vista tradicionalmente considerados como marginales.

Palabras clave: Epistemología; Feminismo; Sandra Harding; Susan Haack; Metáforas.
\end{abstract}

\begin{abstract}
This article deals with the relationship between feminism and science. First, we will offer a panoramic view of the various positions regarding feminist thought and its contribution to the fields of the Theory of Knowledge and the Philosophy of Science; we will present the doctrines of Sandra Harding and Susan Haack, which show the polarization between positions that totally reject the conception and practice of past and present science, considering it absolutely androcentric, in front of those who maintain that science has nothing to do with gender, therefore the only idea of a feminist epistemology is incongruent. Then, finally, we will make an analysis of the above, reaffirming concepts that we consider realistic and rejecting those we deem erroneous or exaggerated, to reach our own conclusions, emphasizing the importance of not overlooking points of view traditionally considered marginal.

Keywords: Epistemology; Feminism; Sandra Harding, Susan Haack, Metaphors.
\end{abstract}




\section{Introducción}

El pensamiento feminista incursionó en la filosofía en lo que podría denominarse un movimiento de los márgenes al centro. Sus primeros campos de interés fueron los campos aplicados, especialmente la ética. Estas áreas son consideradas por muchos filósofos profesionales como la zona periférica del trabajo filosófico central, caracterizando a este por un grado más alto de abstracción de la realidad material concreta y por sus pretensiones de universalidad (Alcoff y Potter, 1993).

Dado que el feminismo es un movimiento político preocupado por asuntos prácticos, es natural que la filosofía feminista contribuyera al debate público en este campo; así, las áreas más abstractas de la filosofía parecían más bien ajenas a aquellas preocupaciones concretas. Con todo, posteriormente las feministas percibieron la presencia del androcentrismo incluso en las áreas "centrales" de la teoría del conocimiento y la filosofía de la ciencia, por lo que también se abocaron a estas disciplinas.

El trabajo feminista comenzó como una crítica a la tradición, ofreciendo nuevas teorías que desafiaban conceptos y criterios comúnmente aceptados respecto de los objetivos, características y estándares científicos que, por lo demás, ya habían sido cuestionados, entre otros por Kuhn (Arrieta, 2016) y Foucault (Arrieta, 2017). No obstante, dentro del propio pensamiento feminista podemos encontrar toda una gama de pareceres que van desde un rechazo radical a la ciencia tal como se la ha concebido y se viene practicando, urgiendo su refundación, a la idea de que una epistemología feminista es incongruente.

En este artículo analizaremos estas posiciones. Empezaremos con una panorámica de las epistemologías feministas (sección I); continuaremos con la presentación de las teorías de Sandra Harding y Susan Haack, antagónicas entre sí, complementando sus puntos de vista con otras doctrinas (sección II); finalmente, analizaremos el material expuesto y presentaremos nuestras conclusiones (sección III). 


\section{Sección I. Epistemologías feministas}

La epistemología feminista es un nuevo enfoque de la epistemología que resalta la importancia de la comunidad científica como tal y se opone a la visión tradicional que privilegiaba el individualismo, al centrarse en el científico como agente de conocimiento.

Considera que la ciencia debe verse como un conocimiento situado inevitablemente en un contexto social y cultural, en el que predominan los valores androcéntricos; consecuentemente, la ciencia habría sido practicada en concordancia con tales valores, manteniéndolos, lo cual dificulta la incorporación de la mujer en este campo. Destaca la singularidad de mujeres y hombres como seres sexuados, en un determinado contexto histórico, vital y con una fuerte carga de desigualdad en la dimensión del género. Así tenemos que, por ejemplo, Rubí Gómez Campos (2013) afirma que "el objetivo de la teoría feminista es superar la parcialidad masculinizante de las ciencias y la filosofía "neutras"” (p. 84).

Según Sandra Harding (1993), los estudios de la ciencia feministas pueden clasificarse en: (i) empirismo feminista espontáneo, (ii) empirismo feminista sofisticado y (iii) epistemología del punto de vista feminista.

Por su parte, Norma Blazquez Graf (2017) propone "tres principales aproximaciones teóricas, las mismas que en los últimos años han ido atenuando las diferencias que las caracterizaban: la teoría del punto de vista feminista [...] el posmodernismo feminista [...] y el empirismo feminista" (p. 20 ss.).

A grandes rasgos caracterizaremos estas posiciones:

(i) Empirismo feminista espontáneo o empirismo feminista. Mantiene que los rasgos sexistas de la investigación científica pueden eliminarse si se hace una correcta aplicación de las normas científicas y se producen cambios en el lenguaje. Sus representantes identifican hechos que a los científicos hombres se les han pasado por alto, no porque les sean inaccesibles, como sostienen las filósofas del punto de vista, sino porque tales hechos para ser reconocidos 
requieren una teoría que incorpore intereses no científicos, valores, incluso gustos que, probablemente, no perciban los científicos criados en un mundo sexista. Además, ellas destacan el carácter social de la ciencia moderna, que incluye equipos y grupos, comunidades y sociedades, instituciones y gobiernos, lo cual tiene tanto un aspecto positivo, como uno negativo. En cuanto a lo primero, la comunidad científica distribuye en forma eficiente y coherente las tareas de investigación, apoya y escruta los hallazgos y las teorías que los individuos proveen, y proporciona recompensas y castigos; todo ello incentiva a los científicos para avanzar en la frontera de la investigación. Respecto a lo segundo, existe la posibilidad de que la comunidad sea una fuente de prejuicios que impida a los individuos una percepción correcta de los hechos empíricos, ofreciendo incentivos perversos para la complicidad en tales prejuicios; propiciaría, además, la indiferencia de los científicos respecto de necesidades y valores humanos importantes en la determinación de la dirección que habría de seguir tanto la investigación pura como la aplicada.

Por todo ello, es necesario tener en cuenta el carácter social de la investigación científica y su deformación de género. Hay un sesgo persistente vinculado, por ejemplo, con la importancia que se atribuye a diferentes cuestiones de investigación; una prueba es el hecho de que aunque los biólogos ignoraron las estrategias reproductivas de las hembras en infrahumanos, cuando vino la anticoncepción el focus de la intervención farmacéutica fue en las mujeres. Por otro lado, en el tratamiento de la depresión — que es un desorden más frecuente en mujeres-, los fármacos fueron examinados solo en muestras de hombres, debido a que se asumió que las diferencias entre la fisiología del hombre y la mujer eran insignificantes. En los antecedentes cognitivos de estas decisiones acerca de cómo proceder en ciencia hay juicios de valor, algunos de los cuáles pasan por alto los intereses de las mujeres. Con todo, estas feministas empiristas no cuestionan el fin de la ciencia de proporcionar conocimiento objetivo, pero sostienen que frecuentemente las teorías, los programas de investigación y los paradigmas son insensibles a todo lo que no sea una contraevidencia muy fuerte ejercida en formas políticamente efectivas. 
(ii) Empirismo feminista sofisticado. Se construye en oposición parcial a las teorías del punto de vista feminista, a los argumentos feministas radicales que exaltan lo femenino y esencializan la "experiencia de la mujer", y al empirismo prefeminista. Con todo, sus representantes incorporan a sus epistemologías elementos que también aparecen en las explicaciones del punto de vista. Sostienen que las hipótesis se convierten en conocimiento cuando se someten al escrutinio de diversas perspectivas, especialmente las de aquellos con creencias y valores diferentes. Helen Longino (1993) afirma que "el conocimiento científico se construye no por individuos que aplican un método al material a ser conocido, sino por individuos que interactúan unos con otros en formas que modifican sus observaciones, teorías e hipótesis" (p. 111) ${ }^{1 *}$; además, aún en ausencia de un consenso respecto de lo que es importante, diferentes subcomunidades — dentro de una más extensa comunidad científica - se pueden interesar en diversas relaciones, descripciones de objetos o modelos y resultar igualmente adecuadas, proporcionando conocimiento "en el sentido de una habilidad para dirigir nuestras interacciones e intervenciones” (Longino, 1993, p. 115).

(iii) Epistemología del punto de vista feminista. Esta teoría sostiene que hay ciertos hechos relevantes a la valoración de las teorías científicas que son detectables desde ciertos puntos de vista o posiciones; así, afirma que el punto de vista femenino y el de los oprimidos tiene una perspectiva parcial y menos perversa que la del poder dominante y que por lo tanto debe privilegiarse. Harding (1993) señala:

La historia intelectual de la teoría del punto de vista feminista es convencionalmente trazada hasta las reflexiones de Hegel sobre qué puede ser conocido acerca de la relación amo/esclavo desde el punto de vista de la vida del esclavo vs. la vida del amo y a la manera en que Marx, Engels y Lukacs subsecuentemente desarrollaron esta visión en el "punto de vista del proletariado" (pp. 53 ss.).

${ }^{*}$ La traducción de esta cita y de todas las siguientes que provienen de textos en inglés ha sido hecha por la autora, igualmente los títulos de los libros referidos. 
Harding destaca las diferencias entre el estilo cognitivo masculino: abstracto, teórico, analítico cuantitativo, deductivo, orientado hacia el control y el dominio; y el estilo cognitivo femenino: concreto, práctico, sintético, cualitativo, intuitivo, orientado hacia el cuidado. De ello se sigue que ciertos hechos no pueden ser detectados por los hombres. Típicamente son hechos difíciles de cuantificar, o incluso de describir en vocabularios ordinarios o científicos, ya que se trata de hechos acerca de los efectos a largo término de la opresión, subordinación, discriminación, estereotipos y se tiene que vivir la posición inferior para realmente detectar lo relevante.

En general, los teóricos del punto de vista no alegan que los hechos físicos o químicos sean errados porque sus hallazgos no presten atención al punto de vista de la mujer u otro marginalizado, pero sí han percibido en la biología un sesgo androcéntrico. Por ejemplo, los sociobiologistas enfatizaron las estrategias de emparejamiento del macho en especies no humanas y no notaron las estrategias femeninas, debido a la incapacidad de los biólogos hombres para localizarse en el punto de vista relevante. Entre las aspiraciones de la teoría del punto de vista está la de emancipación, no solo de las mujeres sino de todos los que han sufrido por las muchas fallas de "objetividad" y "desinterés" que la ciencia oficial puede elogiar pero que los científicos realmente están lejos de alcanzar.

(iv)Postmodernismo feminista. Niega la posibilidad de una objetividad carente de rasgos sexistas y la existencia de un punto de vista femenino único que incluya el universo total de mujeres, pues no hay un modo exclusivo de ser mujer, ya sea que nos refiramos al sexo, a un hecho biológico o al género. Por lo tanto, se debe hablar de "las mujeres", cuya existencia y posición se constituye por la confluencia concreta de distintas diferencias (género, clase, etnia, sexualidad, edad, etc.). Básicamente, niegan la existencia de sujetos universales y rechazan las grandes narrativas emancipatorias, entre las que incluyen al feminismo clásico.

Así, Blazquez Graf (2017) señala dos puntos principales de esta teoría: “[...] el rechazo a la categoría analítica de mujer y la fragmentación infinita de perspectivas" (p. 25). Nancy Piedra (2003) menciona una diversidad de 
propuestas posmodernistas que buscan: deconstruir la categoría mujer (Julia Kristeva), cuestionar la construcción de dos categorías tanto para el sexo como para el género (Henrrieta Moore), considerar como determinante las "relaciones de género" (Jane Flax), tomar de la filosofía, la sociología, el psicoanálisis, etc., — todo lo que sea útil para explicar y visibilizar la subordinación de las mujeres(Nancy Frazer). Además, Sheila Benhabid aboga por el desarrollo de una actitud reflexiva del ser-mujer para resignificar, reinterpretar, transgredir e interrumpir la cadena de significados constituida; mientras que Judith Butler considera que ello no funciona y que se requieren más bien re-significaciones transgresoras; $y$, Luce Irigaray propone la exploración del cuerpo y la experiencia del placer sexual de la mujer como bases idóneas para la construcción de una nueva subjetividad femenina, en ruptura con el discurso del logos fálico.

Se introducen también los horizontes poshumanos propiciados por la tecnociencia. Donna Haraway (1995) nos habla de los cyborgs: "un organismo cibernético, un híbrido de máquina y organismo, una criatura de realidad social y también de ficción" (p. 253), un ser fronterizo, igual a simios y mujeres. Su reconocimiento rompería la frontera entre lo animal y lo humano y acabaría con el paradigma dicotómico y jerárquico que, según ella, podría llevarnos al desastre. Igualmente, Rossi Braidotti (2013), a partir de un monismo neospinozista, afirma que en el mundo en el que vivimos no es posible separar la forma naturaleza de la mediación tecnológica; así, aclara que lo poshumano - tal como ella lo entiende - de ninguna manera es enemigo de lo humano, sino más bien su extensión entre las graves contradicciones y los conflictos sociales de los tiempos contemporáneos.

A pesar de toda esta diversidad podría decirse que las autoras mencionadas, en general, aceptan que la ciencia es importante porque promueve un desarrollo emancipador y asume una política científica crítica; niegan entonces posiciones inocentes o irresponsables y tienen una tendencia a reconocer la inutilidad de renunciar al poder y al placer que la tecnociencia pueda procurar. De este modo, el sujeto de conocimiento es un agente corpóreo, heterogéneo y 
multidimensional, un sujeto posmoderno que asume lo inestable, lo múltiple y lo contradictorio.

\section{Sección II. Sandra Harding vs. Susan Haack}

Sandra Harding (1993) reconoce puntos positivos en el empirismo feminista espontáneo: i) explica la producción de resultados de investigaciones sexistas y no sexistas, desafiando mínimamente a la lógica de investigación fundamental - tal como es comprendida en los campos científicos- y a la lógica de explicación, acorde con los estándares de las filosofías de la ciencia dominantes; ii) esta corriente trata de acomodar los proyectos feministas en los estándares prevalecientes de "buena ciencia" y "buena filosofía"; iii) este conservadurismo posibilita que mucha gente pueda captar la importancia de la investigación feminista en biología y las ciencias sociales sin sentirse desleal a los métodos y normas de sus tradiciones de investigación; iv) el empirismo feminista espontáneo exige un mayor rigor al usar estos métodos y seguir estas normas.

Con todo, Harding considera que tal política conservadora entraña sus debilidades. Una de ellas es el rechazo total a dirigirse frontalmente contra las limitaciones de las concepciones dominantes del método y la explicación, así como las formas en que estas concepciones constriñen y distorsionan tanto los resultados como el pensamiento de las investigaciones. La otra debilidad la configuran las ciencias que han sido ciegas a sus propias prácticas y resultados científicos sexistas y androcéntricos. La autora plantea entonces la siguiente interrogante: ¿son las lógicas de investigación y explicación realmente inocentes respecto de esta ceguera, como afirma el empirismo, o son parte de su causa?

Harding propone una "objetividad fuerte", la que "requiere que el sujeto del conocimiento sea colocado en el mismo plano crítico, causal de los objetos de conocimiento" (1993, p. 69). Contradiciendo a quienes piensan que la epistemología del punto de vista se opone a la aspiración de la ciencia a lograr un conocimiento objetivo afirma que, por el contrario, su teoría aumentará y fortalecerá nuestra habilidad para lograr objetividad. 
En tal sentido sostiene que los investigadores, aun siguiendo con máximo rigor las reglas de los métodos tradicionales de investigación, no son capaces de lograr una objetividad fuerte porque tales métodos no han examinado el contexto de descubrimiento, que se considera no racional; de esta forma, no han identificado los deseos sociales, los intereses y los valores que han formado las ciencias. Así, en la ciencia se omite el sujeto y ella se constituye en un reporte descorporizado, libre de valores, de hechos independientes del contexto. Con todo - dice nuestra autora - la ciencia tiene un sujeto, que en la comunidad occidental es un grupo de machos dominantes con un punto de vista que envuelve asunciones y valores basados en actividades masculinas y, dado que la ciencia tradicional no examina este punto de vista, se empobrece la objetividad que la ciencia podría lograr.

Por otro lado, Harding arguye que el punto de vista de este grupo dominante se presenta limitado epistémicamente con respecto del punto de vista de varios grupos marginalizados. Debido a la homogeneidad del grupo dominante es poco probable que se identifiquen las asunciones que comparten, menos incluso si al grupo le beneficia mantenerlas. De esta manera, lo que recomienda es una metodología que tenga como punto de partida el pensamiento desde las vidas de las gentes marginalizadas, afirmando que esto tendrá un mayor poder revelador sobre las asunciones que influyen en la ciencia, creará problemas a ser explicados o agendas de investigación particularmente significativas; en suma, generará más cuestiones críticas y, por lo tanto, menos explicaciones parciales y distorsionadas.

Harding (1993) alude a Dorothy Smith, quien sostiene que la experiencia de las mujeres es la "base" del conocimiento feminista y que tal conocimiento cambiaría la disciplina de la sociología. Smith señala que el trabajo asignado a las mujeres es el cuidado de los cuerpos de hombres, bebés, niños, ancianos, enfermos y los suyos propios, así como de los lugares donde estos cuerpos existen. Esta clase de "trabajo de mujeres", que los hombres no desean, los libera para constituir los grupos gobernantes e imponerse en el mundo de los conceptos abstractos. 
Harding, al afirmar que todos los intentos de conocimiento están socialmente situados y que algunas de estas localizaciones sociales objetivas son mejores que otras como puntos de inicio para proyectos de conocimiento, va en contra de asunciones fundamentales en la visión del mundo científico y el pensamiento occidental; estos toman a la ciencia como su modelo de producción de conocimiento. Así, la teoría de Harding establece una "lógica de descubrimiento" que tiende a maximizar la objetividad de los resultados de la investigación y, por lo tanto, a producir conocimiento que puede ser para la gente marginalizada, y no para el uso exclusivo de los grupos dominantes en sus proyectos de manejar las vidas de esas personas. Con todo, aclara Harding, este comienzo epistemológicamente aventajado para la investigación no garantiza que el investigador pueda maximizar la objetividad en su explicación; estos fundamentos proporcionan solamente un punto de inicio necesario, no suficiente para la maximización de la objetividad.

Uno de los artículos más referidos de esta autora es "Metáforas sexistas en la ciencia: ¿deberían la historia y la filosofía de la ciencia recibir una clasificación ' $X$ '?'. En él, la autora analiza las metáforas creadas para referirse a la naturaleza. Harding (2000) señala:

Los ejemplos de la simbolización de género generalmente se dan en los márgenes, a los lados de textos —en aquellos lugares donde los hablantes revelan las asunciones que tienen sin necesidad de defenderlas-, creencias que esperan compartir con sus audiencias. Veremos que las audiencias de estos textos para esas asunciones la conforman hombres, que los científicos y filósofos son hombres, y que la mejor actividad científica y pensamiento filosófico acerca de la ciencia está para ser modelado sobre las relaciones más misóginas de los hombres con las mujeres — violación, tortura, elegir "amantes", pensar de la naturaleza de la mujer como buena para nada excepto para la maternidad-. (p. 218)

Luego Harding se refiere a una serie de metáforas que se producen en la ciencia moderna, la cual se supone alcanza reflexiones objetivas, universalmente válidas sobre la naturaleza. Para ella, las concepciones de la naturaleza han cambiado con el tiempo y han sido fuertemente influidas por las estrategias 
políticas usadas en batallas identificables históricamente entre los géneros. " $\mathrm{La}$ política de género ha proporcionado recursos para los avances de la ciencia, y la ciencia ha proporcionado recursos para el avance de la dominación masculina" (Harding, 2000, p. 218 ss.).

Harding se refiere, también, al paso del organicismo platónico al organicismo aristotélico. En el primero, el poder activo en el universo se asoció con la madre tierra viva y nutriente; mientras que en el segundo, la actividad fue asociada con la masculinidad y la pasividad con la femineidad. Tal pasividad, unida al criterio de distinción entre animado e inanimado, favoreció la concepción de una tierra femenina simplemente pasiva, materia inerte e indiferente a las exploraciones y explotaciones de su interior.

Señala que el reemplazo del universo alrededor de la tierra por el universo alrededor del sol, realizado por Copérnico, significó también cambiar de un universo centrado en la mujer a un universo centrado en el hombre. La naturaleza, especialmente la tierra, fue vista en forma ambivalente: como una madre amorosa, benevolente, proveedora en un universo ordenado y planeado; $\mathrm{y}$, como salvaje, incontrolable y violenta capaz de producir tormentas, sequías y caos general. Similarmente, la tierra-mujer, concebida como la especial creación de Dios para el cuidado del hombre, devino en un planeta pequeño, externo, que orbita alrededor del sol masculino.

Debemos observar, sin embargo, que en las edades medieval y antigua el trato que se le dio a la mujer no fue el mejor, pese al sitio central otorgado a la tierra — que metafóricamente representaba a la mujer — de acuerdo con Harding. En cuanto a los pensadores renacentistas, nuestra autora señala que mayormente percibieron a la naturaleza como descontrolada e indomable; así, para ilustrar cómo se debe dominar la potencial violencia del destino, Machiavello (1960) dice:

La fortuna es una mujer y es necesario, si tú deseas dominarla, conquistarla por la fuerza; y se puede ver que ella se deja vencer por los audaces en lugar de por los que proceden con frialdad, y, por lo tanto, como una mujer, ella siempre es amiga de los jóvenes porque son menos cautelosos, más feroces, y la dominan con mayor audacia. (p. 121) 
La quiebra del antiguo orden de la sociedad feudal significó un desorden generalizado y se percibió como una amenaza la notoriedad de las mujeres en ese período. En la Reforma protestante, las mujeres fueron muy activas; ejemplo de ello es que, en Inglaterra, el reinado de Elizabeth I fue uno de los más largos de la historia de este país. Todo ello, junto con la falta de una distinción clara entre lo físico y lo social hizo que la imaginación renacentista asociara los desórdenes naturales y sociales con la mujer, a la que vinculó con la brujería y atribuyó métodos de venganza y control en un mundo que era asumido por muchos como animado y orgánico. Y así llegamos a la famosa metáfora de Bacon, cuyo mentor fue Jaime I de Inglaterra, que apoyó fuertemente la legislación antibrujería y antifeminista:

Porque no tienes más que seguir y perseguir a la naturaleza en sus andanzas, y podrás cuando quieras guiarla y conducirla al mismo lugar otra vez. Tampoco debe un hombre tener escrúpulos en entrar y penetrar en aquellos hoyos y rincones, cuando la inquisición de la verdad es todo su objeto, como lo ha mostrado su majestad con su propio ejemplo. (Citado en Harding, 2000, p. 220)

Harding señala que las metáforas iluminan la visión de la naturaleza; sugiere que así como la naturaleza se asemeja a una máquina, las máquinas podrían asemejarse a seres naturales, y que si la naturaleza es vista como una mujer a la que es apropiado violar y torturar, más que como una madre nutricia, se podría concebir que la violación y la tortura son naturales en las relaciones de hombres con mujeres. Entre los posibles usos de la ciencia - dice nuestra autora- hay prácticas que pueden "crear desastres ecológicos, apoyar el militarismo, hacer de la labor humana un trabajo mutilante tanto física como mentalmente, desarrollar formas de control de "otros" — las mujeres, los colonizados, los pobres"(Harding, 2000, p. 221); entonces, retóricamente, se pregunta si estas prácticas son solo malos usos de la ciencia aplicada, o, si constituyen más bien una ciencia distintivamente masculina.

La otra posición que aquí confrontaremos es la de Susan Haack, autora de una interesante propuesta epistemológica: el fundherentismo. Como es sabido, el fundacionalismo busca un fundamento distinto de la creencia para justificar 
esta, mientras que el coherentismo considera que las creencias se justifican simplemente por su coherencia con otras creencias. Su propuesta es "una teoría intermedia que puede superar las dificultades presentadas por estos conocidos rivales" (Haack, 1997, p. 26).

A pesar de considerarse feminista, su posición respecto de una "epistemología feminista" es radical: rechaza esta rúbrica porque no cree que haya ninguna conexión entre feminismo y epistemología. En su artículo "Conocimiento y propaganda. Reflexiones de una vieja feminista" (2003a) confiesa que lo único extremo acerca de su visión política es su disgusto por los extremos; con todo, rechaza el proyecto de una epistemología feminista por razones más de índole epistemológica que política.

Haack recuerda que el feminismo empezó acentuando la humanidad común de hombres y mujeres, y se enfocó en la justicia y la oportunidad, con una preocupación primaria en temas de la teoría social y política que en verdad debían ser tratados. La autora rechaza, en cambio, los nuevos feminismos que enfatizan "el punto de vista de la mujer" y reclaman una revolución para todas las áreas de la filosofía, incluida la epistemología. Encuentra que no tiene sentido hablar de "epistemología feminista", por lo que considera grave la asombrosa variedad de ideas epistemológicas que se dicen "feministas": cuasifundacionalistas, coherentistas, contextualistas, relativistas; algunas que enfatizan los aspectos sociales del conocimiento, la comunidad y otras que subrayan la emoción (subjetiva y personal); hay quienes buscan el cambio de las normas "androcéntricas" por otras "ginocéntricas", etc. Incluso en donde hay un acuerdo aparente, con frecuencia se enmascara el desacuerdo subyacente acerca del significado, que puede variar para cada cual. Este hecho, aunque es reconocido por diversas autoras, entre ellas Sandra Harding, para Haack es más bien perturbador.

Por lo demás —nos dice—, muchas si no todas las ideas defendidas por las "epistemólogas feministas", también lo han sido por quienes no comparten esta etiqueta, por ejemplo George Sanders Pierce, quién criticó el excesivo 
individualismo de Descartes. Aunque no encuentra razones válidas para la calificación de "epistemóloga feminista", señala que la frase "el punto de vista de la mujer" puede interpretarse de dos maneras: como "la forma en que las mujeres ven las cosas", o "sirviendo a los intereses de las mujeres".

Para Haack (2003a), las diferentes habilidades que muestran muchachos y muchachas no implica que tengan formas distintas de conocer. Indica que ha habido diferentes intentos de probar tal aserto, siendo quizás el esfuerzo más importante el plasmado por Mary Field Belenky y otros en Las formas de conocer de la mujer. Lamentablemente, en dicho libro, antes de realizarse las entrevistas, se indicó a los sujetos que estas eran para estudiar "las formas de conocimiento de las mujeres", con lo que no hay seguridad de que las respuestas carezcan de sesgo.

Haack afirma que todo lo que un ser humano tiene que seguir para descubrir cómo son las cosas, es su experiencia sensorial y retrospectiva, así como la teoría explicativa que él o ella idean para acomodarla; además, sostiene que las diferencias en el estilo cognitivo, tal cual las diferencias en la escritura a mano, parece que fueran más individuales que determinadas por el género. Por otro lado, la profusión de temas, a veces incompatibles, propuestos por la "epistemología feminista" habla más bien en contra de la idea de un estilo cognitivo distintivamente femenino. Incluso si lo hubiera, quedaría por demostrar que las "formas de conocer de las mujeres" son superiores en procedimientos de investigación o en estándares más sutiles de justificación en relación con las de los hombres.

Para Haack, las cuestiones de la tradición epistemológica son sumamente difíciles para que cualquiera las responda o las aclare significativamente, sin importar sexo ni género. Son más bien las idiosincrasias individuales y no el "grupo de pensamiento" - tan admirado por algunas feministas - lo que produce cualquier innovación, ya sea filosófica, científica, artística, etc.

En cuanto al privilegio epistémico del que gozarían los oprimidos, perjudicados y marginalizados por su opresión y desventajas, Haack percibe 
que los verdaderamente privilegiados epistémicamente no serían las mujeres occidentales, ricas, bien educadas, blancas, sino los más oprimidos y perjudicados, algunos de los cuales son hombres.

Por otro lado, dado que el conocimiento está en quienes ostentan el poder, difícilmente lo darán a los oprimidos, con lo que limitan sus posibilidades epistemológicas; por lo tanto, no encuentra justificación para la primera interpretación de "el punto de vista de las mujeres" en "cómo ven las mujeres las cosas". En cuanto a la segunda interpretación "sirven a los intereses de las mujeres", se supone que la conexión se hace por medio de la crítica al sexismo en el teorizar científico. Las rutas que presuntamente conectan el feminismo y la epistemología se fusionarían en la asunción, que Haack no acepta, de que el sexismo en el teorizar científico es el resultado de la exclusión de "formas de conocer" femeninas, lo que también rechaza porque no son solamente hombres quienes suscriben estereotipos sexistas, hay mujeres que también lo hacen.

En las ciencias sociales y con menos frecuencia en primatología y otras ramas de la biología, se han dado casos en que científicos hombres han aceptado afirmaciones que no estaban plenamente apoyadas por la evidencia, debido a que en forma acrítica aceptaron ideas estereotipadas de la conducta masculina y femenina. Haack piensa que estos casos deben ser examinados individualmente y por expertos, sin que sea una credencial de experticia el hecho de ser feminista. En cuanto a extender la crítica feminista de sexismo al teorizar científico en todas las áreas de la ciencia, incluyendo la física, la química, etc., no lo considera adecuado. Ahora bien, Haack no acepta que del descubrimiento de sexismo en el teorizar científico se siga la obligación de reconocer consideraciones políticas como formas legítimas de decidir entre teorías; más bien, este descubrimiento nos debería llevar a la conclusión contraria: que la política debería quedar fuera de la ciencia y que la aceptación de una teoría ha de basarse en la garantía y la calidad de la evidencia.

La filósofa inglesa considera que se ha dado un cambio importante: de la vieja visión diferencialista que afirmaba la autoridad epistémica de la ciencia 
en virtud de su peculiar racionalidad y método de investigación objetivo, se ha pasado a un nuevo cinismo en filosofía de la ciencia que la visualiza como una institución social permeada de valores, que privilegia la importancia de la política, el prejuicio y la propaganda, sobre el peso de la evidencia al determinar qué teorías se aceptan. Así, se llega algunas veces a afirmar que la realidad es construida y que verdad es una palabra que debe ser usada entre comillas. En consecuencia, Haack (2003a) diagnostica:

Que el nuevo cinismo en filosofía de la ciencia ha alimentado la ambición de un feminismo nuevo, imperialista, para colonizar la epistemología. Los valores con los cuales la ciencia está permeada — se arguye - , han sido hasta ahora androcéntricos, sexistas, inhóspitos a los intereses de las mujeres. Las críticas feministas del sexismo en el teorizar científico, continúa el argumento, no pueden ser vistas meramente como críticas de mala ciencia; la moraleja a ser sacada es que debemos abandonar la búsqueda quijotesca de una ciencia libre de valores, en favor del objetivo obtenible de una ciencia informada por valores feministas. (p. 11 ss.)

Lo cual, dice nuestra autora, podría aceptarse si se pudiera legitimar la idea de que los valores feministas deberían determinar qué teorías son aceptadas. Así, Haack (2003a) considera dos líneas de argumentación importantes para los nuevos cínicos: subdeterminación y carga de valores.

La subdeterminación avala que las preferencias políticas determinen la elección de la teoría, si es imposible decidir entre dos teorías aceptadas. Para la filósofa inglesa la respuesta adecuada es que a menos que se disponga de evidencia, los científicos harían mejor en suspender el juicio y que el público lego, incluidos los filósofos, no debe aceptar las afirmaciones de los científicos sin pruebas suficientes. La subdeterminación, en tal sentido, no abonaría a mostrar que legítimamente podemos elegir creer en cualquier teoría que se acomode a nuestros propósitos políticos.

La segunda línea de argumentación considera necesario desvanecer los límites entre la ciencia y los valores y, por lo tanto, nuevamente, resultaría 
apropiado permitir que los valores feministas determinen la elección de la teoría. Haack considera que aquí el problema consiste en derivar "debe" de "es", lo cual resulta evidentemente falso si lo expresamos claramente: Proposiciones acerca de qué cosas son deseables o deplorables podrían ser evidencia de que las cosas son o no son.

Otra versión del argumento se fundaría en la afirmación de que es absolutamente imposible excluir los valores "contextuales" (i.e., lo externo, social y político) de la ciencia. Aquí Haack encuentra la falacia de non sequitur. Quizás los científicos no pueden dejar de lado completamente sus prejuicios, pero de allí no se sigue que se deba permitir que los prejuicios determinen la elección de la teoría.

Entre el viejo diferencialismo que exagera las virtudes de la ciencia y el nuevo cinismo que hace igual con sus vicios, la verdad está en el centro. La primera postura se enfoca exclusivamente en lo lógico, la segunda, en los factores sociológicos; una adecuada filosofía de la ciencia —afirma Haackdebe combinar ambos. Igualmente, considera que las ciencias naturales han sido exitosas, pero ello no significa que sean infalibles o perfectas. Lo que debe hacerse es suprimir o al menos minimizar los sesgos debidos a la parcialidad, la política, las modas y las tendencias.

Haack no afirma la falsedad de todos los temas que están bajo la rúbrica "epistemología feminista". Es verdad, por ejemplo, que los investigadores son profunda y ampliamente dependientes entre sí y que algunas veces solo pueden percibir la relevancia de una evidencia por presión política o prejuicios previos. Pero tales verdades no tienen consecuencias radicales; no se sigue, por ejemplo, que la realidad sea como cualquier comunidad epistémica determine que es, o que no sea un asunto objetivo determinar qué evidencia es relevante.

A pesar de conceder con la epistemología feminista en algunos de sus puntos, Haack rechaza de plano la designación "epistemología feminista" porque, además de considerarla poco rigurosa, esta etiqueta transmite la idea de que la investigación debería ser politizada, considerando que ello no solo es 
errado, sino peligroso por el potencial de tiranía que encierra. Así, rechaza de plano la cita de Harding que dice: “[...] el modelo para la buena ciencia debería ser programas de investigación dirigidos por objetivos políticos liberacionales" (1998, p. 98). Nuestra autora afirma que siguiendo este lineamiento se suprimiría la investigación no contaminada por ideas políticas, esto es, la investigación honesta. Por otro lado, se reforzarían los prejuicios sexistas al mantener una visión femenina de las cosas menos racional o lógica que la masculina. En suma, Haack (2003a) concluye que, epistemológicamente, de cumplirse la aspiración de Harding, se acabaría con la integridad de la investigación y la libertad de pensamiento y, consecuentemente, se causaría un daño a la humanidad y ninguna ayuda a la mujer.

\section{Sección III. Análisis y conclusiones}

Examinaremos el tema de las metáforas y luego nos enfocaremos en los desacuerdos más significativos entre Sandra Harding y Helen Haack.

\section{Metáforas de la ciencia y femineidad}

El punto de vista de Harding respecto de las metáforas ha sido ampliamente discutido. Tiene detractores como Alan Soble y Noretta Koertge, quienes no detectan ninguna misoginia en las metáforas mencionadas por Harding, así como defensores como Evelyn Fox Keller.

Respecto de la más comentada, Koertge (1999) coincide con Soble (2000) en que al atribuir a Bacon un llamamiento a la violación de la Naturaleza están proyectando horrores que en realidad no existen. Pero también tiene defensores como la física, bióloga, autora y feminista norteamericana Evelyn Fox Keller. Ella en una entrevista con Bill Moyers (mayo de 1990) hace importantes aportes al tema. Considera que debemos prestar atención al lenguaje de la ciencia, a su funcionamiento y a cómo está presente la ideología en la ciencia; ello a través del tráfico entre el lenguaje ordinario y el técnico, porque el género —entendido como ideas de masculinidad e ideas de femineidad - juega un rol significativo en el lenguaje que los científicos usan para describir su trabajo. 
Keller señala que una de las instituciones de la ciencia más importantes, la Royal Society, se propuso establecer una filosofía masculina. Bacon nos dice - proponía establecer un matrimonio casto y legal entre la mente y la naturaleza; ahora bien, ella considera que el propósito de este matrimonio patriarcal era esclavizar a la naturaleza y a todas sus criaturas y ponerlas al servicio de la mente, reafirmándose los pares tradicionales: mente/esposo - naturaleza/ esposa. Lo característico de la ciencia respecto de su actividad, pensamiento y filosofía, en su sentido más general, era pensar como un hombre.

Esta autora menciona a Joseph Glanvill, quien considera que la verdad es incompatible con la voluntad o la pasión y que las mujeres son fuente de engaño. El asunto no ha cambiado mucho — nos dice Keller - y alude a las palabras de Luke, un joven científico: "Es maravilloso cuando tienes un problema que realmente está resultando. Es como hacer el amor. De repente, tu inconsciente toma el control, y nada puede detenerte. Sabes que estás haciendo que la Vieja Madre Naturaleza se humille y ruegue, y le dices: 'Te tengo, vieja perra'. La tienes justo donde la quieres”.

Cuathémoc Hernández propone pensar al género como un dispositivo de poder "que tiene en su base un dominio inmenso de relaciones de fuerza desiguales" (2017, p. 57); las mismas están camufladas en diferentes relaciones que van de lo económico a lo sexual, perpetuando la desigualdad, la opresión y la discriminación, lo que podríamos decir se hace evidente en el comentario citado en el párrafo anterior.

Keller especifica que la ciencia no nos da la naturaleza, sino su descripción o teorías científicas acerca de ella. En tal descripción está la propia experiencia subjetiva y no hay manera de evitarlo, no somos capaces de ver a la naturaleza desnuda, libre de nuestros propios valores, esperanzas, temores, ansiedades, deseos y objetivos. La interacción con el mundo es intencional y es evidente que los valores ayudarán a guiar el tipo de pregunta que se desea hacer; ya sea que estos valores correspondan a una ideología de género, de clase o de compromiso con el militarismo. 
Creo que no es posible negar que definitivamente exista un "machismo", como diríamos en América Latina, en las expresiones metafóricas que unen la ciencia y la naturaleza, aunque quizás no en las dimensiones que señalan Harding y Keller; de toda forma, podría decirse que esta ideología subyacente podría ser uno de los factores que intervienen en el trato inicuo que se viene dando a la tierra y en la persistente falta de otorgamiento de premios científicos importantes a mujeres relevantes en el campo de las ciencias.

Veamos ahora los desacuerdos más saltantes entre Sandra Harding y Susan Haack:

Existencia de una epistemología feminista. Como hemos visto, mientras Sandra Harding defiende la existencia de una o más epistemologías feministas, Susan Haack considera que tal existencia no es necesaria e incluso resultaría incongruente. Dado que hay un evidente androcentrismo en la ciencia actual que la misma Susan Haack reconoce (2003), consideramos que no es dañino hablar de una o más epistemologías feministas que denuncien este hecho, muestren evidencia del mismo y propongan alternativas para superarlo, todo ello sin desconocer los logros que la práctica androcéntrica de la ciencia ha obtenido. Sería recomendable propiciar encuentros de feministas interesadas en la gnoseología y la epistemología, a fin de establecer una agenda común en donde se evidencien los puntos de acuerdo y quizás se maticen las diferencias.

La objetividad de la ciencia. La objetividad fuerte que propone Harding "requiere que el sujeto de conocimiento sea colocado en el mismo plano crítico y causal de los objetos de conocimiento" (1993, p. 69); esto es, una "fuerte reflexividad" que se enfoque en las creencias no examinadas de los científicos y sus comunidades, que identifique críticamente los deseos, intereses y valores sociohistóricos que han formado las agendas, los contenidos y los resultados de las ciencias, así como la influencia de estas en el resto de los asuntos humanos.

Harding rechaza la sugerencia de conservar la vieja noción de objetividad como libre de valores y asignar al feminismo la tarea de ver cómo conseguirla. Ella entiende, más bien, que es la noción de método científico la que debe ser 
cambiada, incorporando el contexto del descubrimiento. Aquí es conveniente enfocarnos en los conceptos ya aludidos de: contexto de descubrimiento, se elige el problema de investigación y se formulan hipótesis; contexto de justificación, se idean pruebas empíricas, se las crítica y revisa para proceder luego a la publicación; y contexto de aplicación, concerniente a la tecnología, patentes y política. Sin duda en los contextos de descubrimiento y de aplicación entran en juego los valores que han rendido frutos tanto positivos como negativos.

Ahora bien, en el contexto de justificación podemos plantearnos la pregunta factual: ¿no están también presentes?, y la pregunta normativa: ¿deberían estar presentes? Es probable que en muchos casos la respuesta al primer interrogante sea afirmativa, ya que los deseos, creencias y ambiciones de los científicos pueden permear las justificaciones, a pesar o con la complacencia de la comunidad científica. Con todo, ello significaría hacer, como dice Susan Haack, mala ciencia y no debe llevarnos a abandonar el ideal de la objetividad. Noreta Koertge también comparte esta visión: "Deberíamos intentar en todas las formas mantener la política y la religión fuera del laboratorio. Podríamos no siempre tener éxito en hacerlo, pero eso simplemente significa que deberíamos esforzarnos más, no que deberíamos renunciar al intento" (2003, p. 230).

El punto de vista privilegiado epistémicamente de los marginalizados. Harding estaría de acuerdo con Haack en que las vidas de las teóricas del punto de vista no son precisamente las que proporcionarían mejores bases para ciertas clases de conocimientos. Sin negar que sus propias vidas pueden ofrecer importantes recursos para tales proyectos, ellas sostienen que vidas de mujeres diferentes (algunas veces opuestas) no solo pueden proporcionar tales recursos, sino que aumentan su habilidad para comprender formas distorsionadas de conceptualizar la política, la resistencia, la comunidad y otras nociones clave de las ciencias sociales y de la historia de los grupos dominantes. Harding (1993) menciona los trabajos de Dorothy Smith — quien parte de la perspectiva de nativas canadienses-, de Patricia Hill Collins — que tiene como pensamiento inicial las vidas de las mujeres afroamericanas pobres y en algunos casos analfabetas-, 
de Betina Aptheker - que encuentra valioso iniciar investigaciones desde las vidas cotidianas de mujeres sobrevivientes del holocausto, trabajadoras chicanas de fábricas de conservas, lesbianas mayores, mujeres afroamericanas en la esclavitud y japonesas sobrevivientes de campos de concentración-, en fin, grupos de mujeres con experiencias muy diferentes de las suyas.

Ahora bien, como señala Bat-Ami Bar On (1993), atribuir privilegio epistémico a sujetos socialmente marginalizados no es una innovación feminista. La idea proviene de la Nueva Izquierda, de quienes la tomaron las feministas de la Segunda Ola. Aunque rechazaron la idea de Marx de atribuir privilegio epistémico solo al proletariado, atribuían una ventaja epistémica a los sujetos localizados en los márgenes sociales sobre los localizados en el centro social. Con todo, Bat-Ami Bar On explica que para Marx la sola marginalidad no es una condición necesaria y suficiente para conceder el privilegio epistémico al proletariado; se requiere, además, que esté en el centro de la producción capitalista, que sea "la fuerza creativa viviente de la producción, de la cual se apropia la clase capitalista y la transforma en el capital que da poder a quienes lo poseen" (1993, p. 86).

La explotación del proletariado por la clase capitalista provoca la alienación y el empobrecimiento material de los primeros, manteniendo el poder de los segundos. Así, para Bat-Ami Bar On (1993) la aplicación correcta de este modelo exigiría la identificación de algún sistema social en el cual las mujeres son centrales como un género o clase de sexo y son socialmente marginales debido a esta centralidad.

Sea como fuere, el privilegio epistémico de los marginalizados podría aplicarse en el contexto del descubrimiento, porque al momento de elegir problemas, su posición especial les permitiría sugerir investigaciones que quienes no están en su lugar pasan por alto y que sin duda son importantes. En el contexto de la justificación, pareciera que los marginalizados —al menos en su mayoríaestán más bien en desventaja, porque carecen de los conocimientos que les permitirían entrar a las comunidades científicas y difícilmente los obtendrán de quienes ostentan el poder. 
En cuanto al contexto de la aplicación, un mínimo de justicia social requeriría tomar en consideración el punto de vista de los marginalizados, a fin de que los descubrimientos científicos lejos de ahondar las injusticias pudieran suprimirlas o, al menos, paliarlas.

La politización de la ciencia. Para Haack (2003a) la investigación honesta busca la verdad y cualquier otra cosa es propaganda. Consecuentemente, rechaza de plano las aserciones de feministas como Sandra Harding, Linda Alcoff y Elizabeth Gross que afirman que inevitablemente hay una conexión entre la verdad y la epistemología con la política.

Con todo, ya Foucault (Arrieta, 2017) nos advertía de la perversidad del poder y de su unión con el saber en un nexo social: el poder valida ciertas clases de conocimiento al promover determinadas narrativas y silenciar otras; así, el conocimiento es una fuente de poder porque confiere posición social y técnicas de control social. En los contextos del descubrimiento y de la aplicación, los objetivos políticos del feminismo sin duda pueden y creemos que deben aplicarse. En los de la justificación concordamos con Haack en que la politización tendría resultados negativos que, finalmente, impedirían alcanzar conocimientos "útiles", ya que estarían sesgados. Igualmente, declarar como verdadera una de dos teorías carentes de demostración definitiva para favorecer una determinada posición política no es honesto y, por ende, no debería hacerse; convendría seguir con las investigaciones hasta lograr un resultado claro.

Norma Blasquez Graf (2011) también aspira a la construcción de una ciencia menos jerárquica, que dé cabida a nuevos tópicos de investigación y permita diversas formas de pensamiento provenientes de ópticas diversas tanto de la naturaleza como de la sociedad.

Evelyn Fox Keller, en su entrevista con Moyers (1990), también subraya el inmenso rol que la ciencia juega en nuestros días y juzga que ella podría ser redirigida, implementándose cambios en la forma en que se hace la ciencia y en la dirección en que se mueve. Keller (1983) destaca el pensamiento de Barbara McClintock, de quien escribió su biografía: Un sentimiento por el 
organismo. Esta eminente bióloga sostenía que no se puede hacer una buena investigación sin tener un sentimiento, una empatía con el organismo que se está estudiando; obviamente esta es una idea muy diferente del acto investigativo tradicionalmente concebido como una batalla, lucha, estado de oposición. Ahora bien, estrictamente, la empatía no es una capacidad exclusivamente femenina, aunque por haberla identificado así, se la haya excluido de la ciencia. En este caso, como en muchos otros de la epistemología feminista, los límites de su aplicación están por verse, pero creo que es un aporte real su lucha por incorporar a la ciencia voces no oídas y buscar rectificar situaciones de opresión, exclusión, explotación, falta de reconocimiento, etc., por medio de una comprensión más extensiva de la ciencia y una aplicación más humana de la misma.

\section{Conclusiones}

El empirismo feminista espontáneo cumplió con la función de denunciar la existencia de un sesgo sexista en perjuicio de la mujer, del cual no se había hablado consistentemente antes. Su ventaja estriba en que al no declarar un rompimiento con la ciencia tradicional, no le es exigible que diseñe de una manera completa y acabada cómo habría de ser la nueva ciencia, ni tampoco resultados que superen a la ciencia tradicional. De esta manera, el empirismo feminista sofisticado profundiza el problema denunciado por el anterior empirismo y sus teorías muestran interesantes desarrollos en cuanto a la incorporación de valores en la ciencia, sin que sean concluyentes.

La epistemología del punto de vista feminista, al incorporar perspectivas, especialmente de los marginalizados, puede enriquecer el panorama científico, sobre todo en cuanto a problemas a ser investigados y a aplicaciones de la ciencia. El reconocimiento de varias epistemologías feministas enriquecería el panorama epistemológico general, aportando diferentes perspectivas que en gran medida podrían compatibilizarse. El punto de vista epistemológicamente privilegiado de los marginalizados puede aplicarse al contexto del descubrimiento y debe aplicarse al de la investigación. 
En tal sentido, es importante tomar en cuenta los sesgos que puede tener el sujeto cognoscente para lograr una mayor objetividad en la ciencia (objetivismo fuerte de Sandra Harding), dado que ese conocimiento es atingente a los resultados de la investigación. Ahora bien, tal consideración precisaría de cánones para examinar con propiedad dichos sesgos y no eximiría de cumplir con las exigencias canónicas de la ciencia tradicional.

En cuanto a las metáforas que unen la naturaleza a la mujer, las que señalan Harding y Keller obviamente son perjudiciales para la condición femenina, pese a esfuerzos como los de Alan Soble por defender a Bacon. Aunque de ellas no se sigue necesariamente la fuerza destructiva que les atribuye Harding, convendría la invención de nuevas metáforas más amigables a las mujeres, lo cual es una tarea que podrían asumir tanto las mujeres como los hombres.

La politización de la ciencia es evidente en algunos casos y se evidencia en la elección de temas a investigarse. Se apoya fuertemente muchas líneas de investigación que responden a intereses de quienes ostentan el poder político o económico, mientras se ignoran otras. Por ejemplo, mientras el SIDA estuvo más o menos confinado a homosexuales, prostitutas, latinos y negros no se le prestó mucha atención, lo que sí ocurrió cuando se extendió a los blancos.

Concordamos con Haack en que ello no debería ser así y que se debe luchar por evitarlo. Creo que es evidente que en el mundo actual la ciencia atraviesa nuestras vidas, no importando que la admiremos o la temamos, que adoptemos sus implicaciones y aplicaciones o las rechacemos. La ciencia penetra nuestras formas de pensar en un número mayor de formas de las que habitualmente reconocemos o deseamos y es razonable pensar que las ideas, emociones, valores y ambiciones humanas también entran en la práctica y la teorización científica. Así, un tema importante — en el que inciden las epistemólogas feministas - es si sus efectos son positivos o negativos, si nos ayuda a conseguir nuestras metas y lograr nuestros sueños o si nos expone a condiciones inhumanas. En síntesis, si sirve para el florecimiento de la humanidad o si se convierte en un despliegue malvado de poder. 
De esta forma, nuevos escenarios nos permiten una mayor comprensión de la interrelación humano-tecnológica propia del mundo en el que vivimos - v. g. los cyborgs de Donna Haraway y los sujetos nómadas de Rossi Braidottiy ello nos enfrenta a nuevas concepciones del ser humano; sin embargo, los reclamos feministas tienen plena vigencia, pues aún persisten injusticias contra la mujer.

Finalmente, en el campo de la ciencia y de la filosofía de la ciencia - como en muchos otros habitualmente vistos como "masculinos"- las mujeres van ganando presencia y sus aportes son paulatinamente reconocidos, posicionándose un buen número de ellas en el centro; con todo, la lucha feminista por la igualdad de derechos, oportunidades y reconocimientos sigue siendo vigente para muchas y muchos marginalizados, de allí la importancia de seguir investigando estos temas.

\section{Referencias bibliográficas}

Alcoff, L. \& Potter, E. (1993). Feminist Epistemologies. Nueva York: Routledge.

Arrieta, T. (2016). Paradigmas de la ciencia, utopías y antiutopías. En T. Arrieta. Perfiles filosóficos (pp. 9-63). Arequipa: Editorial UNSA.

Arrieta, T. (2017). Valores tradicionales en Michel Foucault y su influencia en las epistemologías feministas. En T. Arrieta, W. Tapia, J.A. Valdivia \& N. Rosado (Eds.), Actas Congreso Internacional de Filosofia de la Ciencia y la Tecnología (pp. 19-31). Arequipa: Editorial UNSA.

Blazquez Graf, N. (2011). El retorno de las brujas. Incorporación, aportaciones y críticas de las mujeres a la ciencia. Ciudad de México: UNAM, Centro de Investigaciones Interdisciplinarias en Ciencias y Humanidades.

Blazquez Graf, N. (2017). Epistemología feminista: temas centrales. En: K. da Rosa, M. Caetano \& P. Almeida (Eds.), Gênero e sexualidade: interseccções necesárias ã produção de conhecimentos (pp. 12-31). Campiña Grande: Realize.

Bar On, Bat-Ami. (1993). Marginality and Epistemic Privilege. En L. Alcoff \& E. Potter. Feminist Epistemologies (pp. 83-100). Nueva York: Routledge. 
Belenky. M. F. et ál. (1998). Women's ways of knowing: The development of self, voice and mind. Nueva York: Basic.

Braidotti, R. (2013). Lo posthumano. Madrid: GEDISA.

Gómez, R. (2013). El feminismo es un humanismo. Barcelona: Anthropos.

Haack, S. (1997). Evidencia e investigación. Hacia la reconstrucción en epistemología. Barcelona: Tecnos.

Haack, S. (2003). After My Own Heart. Dorothy Sayers's Feminism. En C. Pinnick, N. Koertge \& R. Almeder (Eds.), Scrutinizing Feminist Epistemology (pp. 244-251). Nueva Jersey: Rutgers University Press.

Haack, S. (2003a). Knowledge and Propaganda. Reflections of an Old Feminist. En C. Pinnick, Koertge \& R. Almeder (Eds.), Scrutinizing Feminist Epistemology. An Examination of Gender in Science (pp. 7-29). Nueva Jersey: Rutgers University Press.

Haraway, D. (1995). Ciencia, cyborgs y mujeres. La reinvención de la naturaleza. Madrid: Cátedra.

Harding, S. (1993). Rethinking Standpoint Epistemology: 'What Is Strong Objectivity'? En L. Alcoff, \& E. Potter (Eds.), Feminist Epistemologies (pp. 49-82). Nueva York: Routledge.

Harding, S. (1998). Whose Science? Whose Knowledge? Thinking from women's lives. Nueva York: Cornell University.

Harding, S. (2000). Sexist Metaphors in Science: 'Should the History and Philosophy of Science Be X-Rated'. En T. Schick Jr. Readings in Philosophy of Science. From Positivism to Postmodernism (pp. 218221). California: Mountain Blue.

Hernández, C. (2017). El dispositivo sexo-género. Guanajuato: Cátedra de Filosofía y Literatura José Revueltas.

Keller, E. F. (1983). A feeling for the organism: the life and work of Barbara McClintock. Nueva York: Freeman.

Koertge, N. (1999). na conversación con Noreta Koertge. En Daniel Greenberger, Wolfgang L. Reiter \& Anton Zeilinger (Eds.), Epistemological and 
Experimental Perspectives on Quantum Physics (Vienna Circle Institute Yearbook \#7) (pp. 279-301). Dordrecht: Kluwer Academic Publishers.

Koertge, N. (2003). Feminist Values in Science. En C. Pinnick, N. Koertge \& R. Almeder (Eds.), Scrutinizing Feminist Epistemology. An Examination of Gender in Science (pp. 222-233). Nueva Jersey: Rutgers University Press.

Longino, H. (1993). Subjects, Power, and Knowledge. En: L. Alcoff y E. Potter (Eds.), Feminist Epistemologies (pp. 101-120). Nueva York: Routledge.

Machiavello, N. 1960. El príncipe. Buenos Aires: Editorial Sopena.

Moyers, B. (1990). Entrevista: Bill Moyers. Evelyn Fox Keller: The Gendered Language of Science. Mayo. Recuperado de http://billmoyers.com/ content/evelyn-fox-keller

Piedra, N. (2003). Feminismo y postmodernidad: entre el ser para sí o el ser para otros. Ciencias Sociales, III-IV (101-102), 43-55.

Soble, A. (2000). "In Defense of Bacon”. En T. Schick, Readings in Philosophy of Science. From Positivism to Postmodernism (pp. 222-230). California: Mountain Blue. 